EPJ Web of Conferences 19, 09005 (2012)

DOI: $10.1051 /$ epjconf/20121909005

(C) Owned by the authors, published by EDP Sciences, 2012

\title{
Investigating the Milky Way with the RAVE chemical catalog
}

\author{
C. Boeche ${ }^{1, a}$, A. Siebert ${ }^{2}$ and M. Steinmetz ${ }^{1}$ \\ ${ }^{1}$ Leibniz Institut für Astrophysik Potsdam (AIP), An der Sternwarte 16, 14482 Potsdam, \\ Germany \\ ${ }^{2}$ Observatoire de Strasbourg, Université de Strasbourg, CNRS 11 rue de l'université, \\ 67000 Strasbourg, France
}

\begin{abstract}
RAVE is a large spectroscopic survey of the Milky Way, aiming at observing up to one million stars by 2012 and at obtaining radial velocities and atmosphere parameters (see Steinmetz et al. [6], Zwitter et al. [7], Siebert et al. [5]). Despite to their medium resolution $(\mathrm{R} \sim 7500)$, and the small wavelength window (8410-8795 ̊̊) RAVE spectra are suitable to perform a chemical abundance analysis. For 234,227 spectra (to date April 2011) we derived abundance estimates for up to 7 elements, which makes RAVE the largest chemical abundances database existing today. We present here the radial chemical gradients of the Milky Way by using RAVE data.
\end{abstract}

\section{THE RAVE CHEMICAL PROCESSING PIPELINE}

In order to obtain the chemical abundances from the RAVE spectra, we developed a processing pipeline. It is a composition of Perl and Yorick routines which measures abundances by fitting normalized spectra with a model spectrum. The model is reconstructed line by line by using the curves of growth of the lines provided by MOOG, a LTE line analysis software (Sneden, [8]). The pipeline renders reliable chemical abundances of up to 7 elements: $\mathrm{Mg}, \mathrm{Al}, \mathrm{Si}, \mathrm{Ca}, \mathrm{Ti}, \mathrm{Fe}$. It relies on the stellar parameters like temperature, gravity and metallicity $(\mathrm{T}, \log \mathrm{g},[\mathrm{M} / \mathrm{H}])$ provided by RAVE (Siebert et al., [5]), on a list of 604 absorption lines and on models of stellar atmospheres (Castelli \& Kurucz, [2]). Expected errors: for $\mathrm{S} / \mathrm{N}>40$ we expect abundances errors of $\sim 0.2$ dex which can grow up to $\sim 0.3$ dex for spectra with $20>\mathrm{S} / \mathrm{N}>40$.

\section{CHEMICAL GRADIENTS ALONG THE GALACTIC RADIUS}

We measured the chemical gradient along the Galactic radius $R$ for the elements $\mathrm{Mg}, \mathrm{Al}, \mathrm{Si}, \mathrm{Ti}$, Fe for a sample of 26,633 dwarf stars of the RAVE chemical catalog (we discuss here only $\mathrm{Fe}$ since the other elements follow the trend). Thanks to distance estimations (Zwitter et al., [7]) their galactic orbits are computed and the mean galactic radius $R_{w}$ calculated as the weighted average of the galactocentric distance over one orbit. We measured the gradients by using the maximum likelihood method to fit the mode of the stars' distribution on the $\left(R_{w},[\mathrm{Fe} / \mathrm{H}]\right)$ plane. The resulting gradient is $\mathrm{d}([\mathrm{Fe} / \mathrm{H}]) / \mathrm{d} R_{w}=-0.054 \mathrm{dex} / \mathrm{kpc}$ for stars having $\left|Z_{\max }\right|<0.4 \mathrm{kpc}$ (black points in Fig. 1). (|$Z_{\max } \mid$ : maximum altitude above the galactic plane reached by the star along its orbit). Interestingly we found that the gradient become flatter at higher $\left|Z_{\max }\right|: \mathrm{d}([\mathrm{Fe} / \mathrm{H}]) / \mathrm{d} R_{w}=-0.037 \mathrm{dex} / \mathrm{kpc}$ for stars with $0.4<\left|Z_{\text {max }}\right|<0.8 \mathrm{kpc}$ and $\mathrm{d}([\mathrm{Fe} / \mathrm{H}]) / \mathrm{d} R_{w}=+0.006 \mathrm{dex} / \mathrm{kpc}$ for stars with $\left|Z_{\text {max }}\right|>0.8 \mathrm{kpc}$ (light

\footnotetext{
ae-mail: cboeche@aip.de
}

This is an Open Access article distributed under the terms of the Creative Commons Attribution-Noncommercial License 3.0, which permits unrestricted use, distribution, and reproduction in any noncommercial medium, provided the original work is properly cited. 


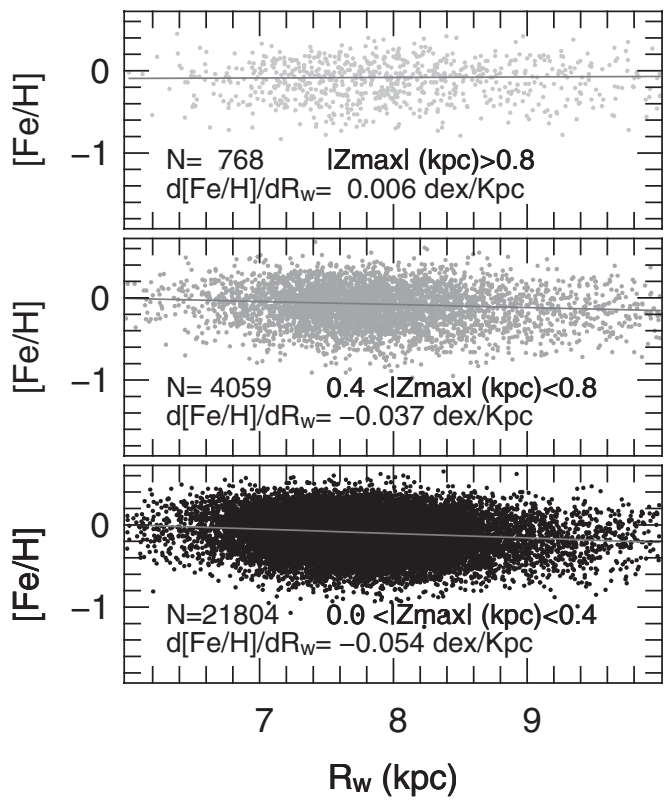

Figure 1. Distributions on the $\left(R_{w},[\mathrm{Fe} / \mathrm{H}]\right)$ plane of three subsamples having different $\left|Z_{\max }\right|$. Into the panels are reported the $\left|Z_{\max }\right|$ ranges and the gradients values.
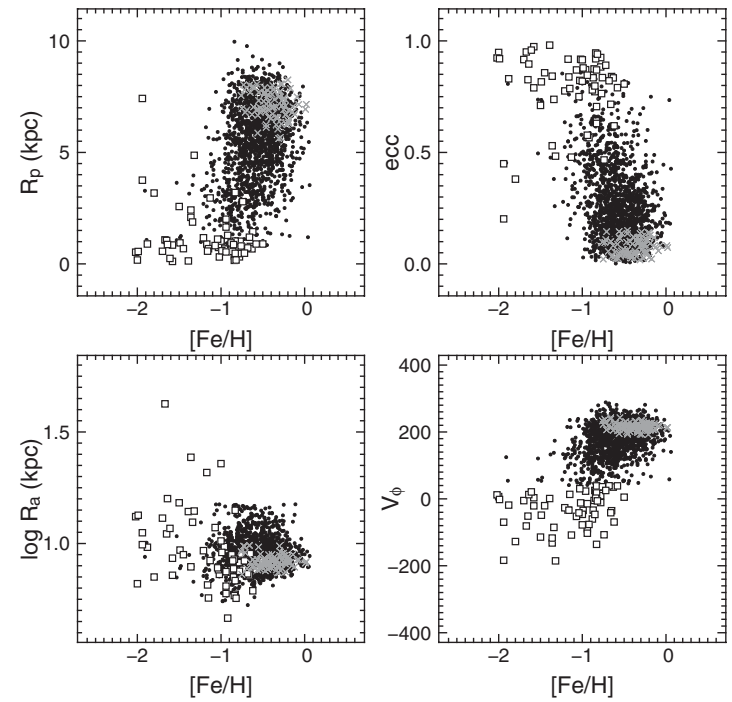

Figure 2. Distribution of the three subsamples in pericenter $\left(\mathbf{R}_{p}\right)$, apocenter $\left(\mathrm{R}_{a}\right)$, eccentricity (ecc) and tangential velocity $\left(\mathrm{V}_{\phi}\right)$ as function of the Iron abundance $[\mathrm{Fe} / \mathrm{H}]$. The symbols identify the thin disk component (gray crosses), the dissipative component (black points) and the accretion component (open squares).

gray points). The gradient for stars with $\left|Z_{\text {max }}\right|<0.4 \mathrm{kpc}$ confirms our present knowledge whereas stars with $\left|Z_{\text {max }}\right|>0.8 \mathrm{kpc}$ raise new questions about their role in the Galactic evolution. An important fraction of such stars have thick disk kinematics and thin disk chemical abundances (these objects were already found by other authors, see Bensby et al. [1], Reddy et al. [4]) and further investigations are needed to identify their origin. 


\section{DISENTANGLING GALACTIC STELLAR POPULATION}

With a sample of 1658 giant RAVE stars with $\mathrm{S} / \mathrm{N}>80$ we repeated the analysis of the pioneering paper by Gratton et al. [3]. We divide the sample in three subsamples: i) dissipative collapse component: stars with $\mathrm{V}_{\phi}>40 \mathrm{~km} / \mathrm{sec}$ and $R_{a}<15 \mathrm{kpc}$. They identified the inner rotating population ii) accretion component: non rotating or counter rotating stars. This population belong to the halo iii) thin disk component: stars whose orbit has low eccentricity $(e)$ and low inclination on the galactic plane and it satisfied the constraints $\sqrt{Z_{\max }^{2}+4 e^{2}}<0.35$. The thin disk component (gray crosses in Fig. 2) has the highest Fe abundance. The dissipative component (black points) reach lower abundances and show $\alpha$-enhancement. The accretion component (open squares) has the lowest Fe abundance and a positive and constant $\alpha$-enhancement. By using the RAVE medium resolution spectra we successfully reproduced results obtained with high resolution spectra but with a sample 100 times larger. Our analysis shows that the criteria can disentangle the accretion component but still the thin and thick disk are blend and they not represent the correct proportion of these two populations (too few thin disk stars with respect to the thick disk). We are searching for new better criteria.

\section{References}

[1] Bensby T. et al., A\&A 410, (2003) 527

[2] Castelli, F., Kurucz, R. L., IAUS, 210, (2003) P.A20C

[3] Gratton R.G. et al., A\&A 406, (2003) 131

[4] Reddy B.E. et al., MNRAS 367, (2006) 1329

[5] Siebert A. and the RAVE collaboration, AJ 141, (2011) 187

[6] Steinmetz M. and the RAVE collaboration, AJ 132, (2006) 1645

[7] Zwitter T. and the RAVE collaboration, AJ 136, (2008) 421

[8] Sneden C. A., Ph.D. thesis, (1973) The University of Texas at Austin 